\title{
FACTORES PSICOSOCIALES EN LOS MIGRANTES DE LA PARADA, NORTE DE SANTANDER
}

\section{PSYCHOSOCIAL FACTORS IN THE MIGRANTS OF LA PARADA, NORTH OF SANTANDER}

\section{Rocío de Belén Contreras Manrique ${ }^{1}$}

\section{Liliana Contreras Manrique ${ }^{2}$}

\section{Olga Lucy Rincon Leal ${ }^{3}$}

Universidad de Pamplona y

Universidad Francisco de Paula Santander

\section{RESUMEN}

En Colombia, y especialmente en la región Norte Santandereana, se presenta un impacto en los factores psicosociales en la zona de frontera, debido a la migración de venezolanos, como consecuencia al éxodo en el país desde el año 2017 y es notorio el impacto de la llegada de venezolanos y colombianos, que habían vivido por décadas en Venezuela, siendo un fenómeno social, psicológico, económico y educativo. En el estudio el objetivo principal fue identificar las características de los factores

\footnotetext{
$1 \quad$ Maestría en prácticas pedagógicas. Universidad de Pamplona (Colombia). orcid.org/0000-0002-4434-0408. rociodebelen@unipamplona.edu.co.rocio_de_belen@yahoo.com.mx

2 Maestría en Orientación. Universidad de Pamplona (Colombia). orcid.org/0000-0002-8586-2093 .lilianacontrerasmanrique@yahoo.com.mx.

$3 \quad$ Maestría en Educación Matemática. Universidad Francisco de Paula. (Colombia). orcid.org/0000-0002-8080496X. olgalucy2702@hotmail.com.
}

psicosociales que influyen en los migrantes a través de la dinámica de movilidad y el impacto en la problemática de frontera en la Parada (N.S). El enfoque cuantitativo, descriptivo, muestreo no probabilístico e intencional con 100 migrantes y la aplicación de un cuestionario de factores psicosociales asociados al bienestar de migrantes de Javier Murillo y Fernando Molero. El análisis de resultado mostrò que el factor de prejuicio percibido por parte del migrante es poco agradable y se encuentran estigmatizados en la zona de frontera por diversas cuestiones económicas y sociales, siendo asistencialistas para poder sobrevivir en la crisis; seguidamente, el segundo factor la discriminación es percibida como delincuentes; en el tercer factor, la identidad de nacionalidad, se reconocen como venezolanos y se le dificulta la economía colombiana. En el cuarto factor, la satisfacción con la vida muestra poca resiliencia 
a un nuevo proyecto de vida; el quinto factor autoestima se encuentran con sentimientos de tristeza, por estar lejos de su familia y por último bienestar material percibido en los migrantes una muestra pequeña reconoce el apoyo de Colombia. Conclusiones: los migrantes se enfrentan a diversas problemáticas sociales como la aculturación, integración, discriminación y aceptación es importante el apoyo psicosocial a los migrantes a travès de un grupo interdisciplinario para fortalecer el proyecto de vida.

\section{PALABRAS CLAVE}

Factor de Prejuicio percibido, factor de la discriminación percibida, factor de la identidad de nacionalidad, factor de la Satisfacción con la vida, factor de Autoestima y bienestar, migración.

\section{ABSTRACT}

In Colombia, and especially in the North Santandereana region, there is an impact on psychosocial factors in the border area, due to the migration of Venezuelans, because of the exodus in the country since 2017 and the impact of the arrival of Venezuelans and Colombians, who had lived for decades in Venezuela, being a social, psychological, economic, and educational phenomenon. The main objective of the study was to identify the characteristics of the psychosocial factors that influence migrants through the dynamics of mobility and the impact on the border problem in La Parada (N.S). The quantitative, descriptive approach, nonprobabilistic and intentional sampling with 100 migrants and the application of a questionnaire of psychosocial factors associated with the wellbeing of migrants by Javier Murillo and Fernando Molero. The analysis of the results showed that the factor of prejudice perceived by the migrant is not very pleasant and they are stigmatized in the border area due to various economic and social issues, being welfare supporters to survive in the crisis; next, the second factor, discrimination is perceived as criminals; In the third factor, the identity of nationality, they recognize themselves as Venezuelans and the Colombian economy becomes difficult for them. In the fourth factor, satisfaction with life shows little resilience to a new life project; The fifth self-esteem factor is feelings of sadness, for being away from their family and, finally, material well-being perceived in the migrants. A small sample recognizes the support of Colombia. Conclusions: migrants face various social problems such as acculturation, integration, discrimination, and acceptance. Psychosocial support for migrants through an interdisciplinary group is important to strengthen the life project.

\section{KEYWORDS}

microsoft teams; moodle; zoom; google classroom; digital libraries; google play.

\section{INTRODUCCION}

En Colombia y en especial en la región Norte Santandereana, se ha presentado un impacto social, educación, económico y psicológico, debido a la migración de venezolanos, siendo la frontera el centro de represamiento de migrantes; por tal motivo, se ha iniciado un éxodo en el país desde el año 2017; sin embargo, es notorio el impacto de la llegada de venezolanos y colombianos, que habían vivido por décadas en Venezuela y como consecuencia a un fenómeno social, por el efecto del régimen de la revolución Bolivariana.

A continuación, se menciona, los datos que genero la oficina de migración Colombiana y se tienen reportados 40.000 Venezolanos viviendo legalmente y calcula que estén 60.000 irregularmente; también se dio a conocer por el periódico del tiempo y que revela que son 900.000 los venezolanos e incluyendo los que tienen doble nacionalidad (el 1,8 por ciento de nuestra población), los que han llegado al país en los últimos 20 años, donde se resalta la crisis 
social, económico y político del país venezolano que se encuentra como frontera del Norte de Santander.

Actualmente en el 2020, Las cifras en la base de datos migración en el corte del 30 de septiembre, en el pais hay aproximadamente 1.715.831 migrantes y que día a día siguen llegando más venezolanos en el tiempo de la pandemia del COVI 19 y de crisis socioeconòmica.

Relevante, en la investigación, la identificación de las características de los factores psicosociales que influyen en los migrantes a través de la dinámica de movilidad y el impacto en la problemática social; por lo tanto, el objetivo específico es la categorización de los factores psicosociales que influyen en los migrantes descubriendo de la situación actual en la frontera y, por último, se describen los factores psicosociales que influyen en los migrantes como indicador en la situación de frontera. Un trabajo entre la Secretaría de Fronteras y Cooperación Internacional de Norte de Santander, la Alcaldía de Villa del Rosario, ACNUR, OIM y las autoridades policiales, se logró despejar a inmigrantes venezolanos del sector de la Parada. Como consecuencia a esta problemática se utilizo como herramienta de estudio un cuestionario de factores psicosociales asociados al bienestar de migrantes de Javier Murillo y Fernando Molero.

A consecuencia de la migración en el corregimiento de la Parada. Se tiene que 880 personas que estaban instaladas en cambuches en la autopista de Villa Rosario en el corregimiento de la parada hasta llegar al Puente Internacional Simón Bolívar, donde fueron ubicadas en el Centro de atención integral de tienditas y el hotel Villa Antigua, de Villa del Rosario, en el proceso y espera de la autorización para ingresar a Venezuela.
De la tal manera, como consecuencia a la crisis económica y social "Decenas de migrantes se habían instalado en un lote propiedad de la policía metropolitana, en malas condiciones de salubridad y saneamiento básico que estaba colocando en peligro la salud de cerca de 3 mil personas que alcanzaron a estar en este lugar, expresó. el secretario de fronteras y Cooperación Internacional de Norte de Santander.

Es importante en base a lo estudiado con las características de los factores psicosociales que influyen en los migrantes de la parada realizar un apoyo interdisciplinario para centranse en la resiliencia de los migrantes proyectándolos a un buen fuuro de bienestar, salud, educación, unión familiar y paz.

A continuaciòn se destacan algunos estudio que sirven de apoyo a la investigaciòn:

Sánchez Isaza \& Cataño Ordoñez, (2016). Colombia con problemas sociales como es la migración de familias, lo que hace necesario la caracterización del factor de riesgo total en las familias para su intervención. En este estudio cuantitativo descriptivo transversal, se seleccionaron 70 familias migrantes constituidas por 344 individuos, que viven en Soledad Atlántico. Discusión: la problemática que viene afectando a las poblaciones rurales, en las familias migrantes, los problemas de infraestructura de las viviendas y la situación socioeconómica, siendo destacado la salud o la atención en la salud y que en algunos momentos se ha vulnerado sus derechos al migrante Adrián, V. A., \& Sagredo, E. P. (2010). Factores de riesgo y protección en madres migrantes transnacionales. Resulto primordial que el médico evalué en la historia de la mujer los diferentes factores de origen biológico, psicológico y social, esto es especialmente cierto en el caso de las madres transnacionales, donde una estrategia de afrontamiento disfuncional de sus estresores psicosociales que puede ocasionar patologías, resaltando las del orden 
psicológico como los trastornos del ánimo, de ansiedad o psicosomáticos.

Contreras-Manrique, R. de B., ContrerasManrique, L.., Ovalle-Lizcano, T. V.., EspinosaBohórquez, L. M. Valero-Bencardino, J. ., Coronel-Peñuela, D. L. ., \& Cabrera-Reyes, A. (2020). Descripción de los problemas sociales influyentes en la transición moral en una sociedad cambiante a través del enfoque cuantitativo. Los autores, analizaron las diversas crisis que ha tratado de sobrevivir como la sobrepoblación, las pandemias que reducen la durabilidad, que inducirán al derecho a dar una vuelta y acoplarse a la civilización, llegará el tiempo, que el deber del estado ya no será asegurar la vida sino la supervivencia, en consecuencia, a un control de integrantes en la familia; siendo la moral y el derecho piezas moldeables a las necesidades de la sociedad.

García Flórez, (2013). Analizó los patrones de migración en Colombia desde la perspectiva de la teoría de redes. El análisis está dividido en dos partes: la primera es la formulación de un modelo basado en la teoría de redes que permita dar cuenta del fenómeno migratorio, de sus rasgos en el marco de referencia para el análisis. Se encontró que Bogotá y Valle del Cauca concentran la mayor recepción de migrantes, siendo importante en los departamentos como expulsores.

Albuja, Sebastián. Albuja, S., \& Ceballos, M. (2010). Desplazamiento urbano y migración en Colombia. El artículo discutió los datos de desplazamiento forzado en Colombia, los cuales muestran que $93 \%$ de los desplazados deciden asentarse en áreas urbanas, en particular la ciudad de Bogotá. Los autores analizaron algunas medidas tomadas por el gobierno local de Bogotá para enfrentar las presiones demográficas que origina el fenómeno de los desplazamientos forzados junto con las migraciones del campo a la ciudad.
Contreras-Manrique Liliana, ContrerasManrique Rocío de Belén, Ovalle-Lizcano Tatiana Valentina, Jaime Fernando RincónLeal. (2020). Análisis descriptivo de rasgos de personalidad en relación con el género y la edad de los estudiantes de la universidad de pamplona en la sede villa rosario. Los rasgos de personalidad que influyen en los jóvenes ya sea en su medio ambiente, particularmente en los procesos de socialización con niveles de ansiedad, sentimientos de tristeza o trastorno como la depresión, agresividad que afectan al estudiante en su proceso de aprendizaje e interacción con su comunidad educativa y en la formación profesional.

Fernández Niño, Julián Alfredo y otros. (2018). Necesidades percibidas de salud por los migrantes desde Venezuela en el asentamiento de Villa Caracas en Barranquilla, se representó, el sistema de salud como un gran reto por la economía, las familias colombianas, siendo la ciudad unas zonas de asentamiento por migrantes venezolanos y colombianos de retorno, en condiciones de vulnerabilidad. cuya participación fue de casi 100 personas, resaltando la mayor población en el género femenino. Conclusiones: se destacó las condiciones de vulnerabilidad de los migrantes para el desarrollo de planes de respuesta a nivel local, sobre el saneamiento ambiental en lo que tiene que ver los social y desmejorando el nivel de la salud por causas y la transmisión de enfermedades infecciosas en el ser humano como consecuencia al trabajo que emprendieron como trabajadores sexuales tanto hombres y mujeres para el sostenimiento de las familias inmigrantes.

Deusdad Ayala, B., Moya, E. M., \& Chávez Baray, S. M. (2012). Violencia de género y mujeres migrantes en la frontera: en Texas. La violencia contra las mujeres se manifestó en diferentes ámbitos de la historia en las sociedades. Incluso en las sociedades modernas 
occidentales la violencia de género de forma transversal y afecta mujeres de otros grupos sociales a nivel socioeconómico y cultural. El nivel de estudios y los factores culturales inciden específicamente en cómo se enfrentan y reaccionan las mujeres al maltrato en un nivel menor de cultura. Las mujeres migrantes víctimas de violencia en Estados Unidos tiene una marginalidad y para mitigar la vulnerabilidad - la marginalidad se realizan talleres para favorecer el empoderamiento de estas mujeres que mejoran sus relaciones afectivas.

Clemens, Michael \& Cindy Huang (2019). Los efectos de la migración dependen de la respuesta al dónde, cuándo, cómo y quién. El debate político sobre la migración se ha centrado en las crecientes crisis de refugiados que ha incluido el régimen político en las naciones. En efecto, la migración tiene un impacto que depende del contexto y de las opciones políticas y económicas de cada país. De manera similar, la migración puede desestabilizar la economía en el país que recibe a los migrantes, que pueden mejorar o declinar la calidad de los servicios públicos en un país.

Camargo, Astrid León (2018). El impacto de la migración venezolana en el empleo en Colombia la migración es un fenómeno reciente que ha surgió por régimen político y económica del nuevo milenio y origino un cambio en la economía venezolana a una economía débil. La situación forzó a la población a abandonar su país para buscar oportunidades en otros países, principalmente en Colombia que fue de gran preferencia de los migrantes venezolanos; un efecto de la migración, ya que la población venezolana no estaba acostumbrada a emigrar y la colombiana a recibir migrantes. Lo anterior influye en el contexto económico de Colombia, especialmente en el empleo, por tanto, es un desafío que exige profundizar en el estudio del impacto de la migración venezolana en el mercado laboral, se hace necesario implementar estrategias que faciliten insertar a la población migrante en la economía colombiana para que no se conviertan en personas asistencialistas.

Tovar Cuevas, Luis Miguel, \& Victoria Paredes, María Teresa, \& Tovar Cuevas, José Rafael, \& Troncoso Marmolejo, Gilberto, \& Pereira Laverde, Fernando (2018). Factores asociados a la probabilidad de emprendimiento en migrantes colombianos que retornan a Colombia. En la investigación, se identificó factores asociados al social cuando un colombiano retorna a su país sea emprendedor. La información de la Global Entrepreneurship Monitor (GEM) Colombia 2012 fue utilizada para ajustar modelos de regresión binaria, estos modelos de referencia benefician la economía de un país ya que poseen habilidades de emprendimiento, con capacidades cognitivas que fortalecen a través de la educación superior, con capacidad monetaria en los ahorros o capital estando fuera de Colombia para relacionarse con los socios o proveedores, siendo las variables principales en un empresario. En efecto se puede dar un valor agregado ya que son personas emprendedoras que van a generar empleo en una ciudad o país aportando beneficios a la economía y a la bolsa de empleo en una comunidad.

Pineda G, Esther y Ávila, Keymer. (2019). La situación política, económica y social de Colombia durante décadas motivó la migración de sus ciudadanos hacia Venezuela y en la actualidad se presenta el retorno de la mitad de colombiano y venezolanos a Colombia. En la cual da un impacto de un país receptor de inmigrantes. Como consecuencia a la crisis económica, política, social desde el año 2015 se evidencio un incremento de este fenómeno como consecuencia de la profundización de la crisis política, económica y social experimentada por la sociedad venezolana y la pauperización de las condiciones de vida de gran parte de la población; esta situación ha convertido a Colombia en la opción migratoria más cercana 
y accesible para los venezolanos en busca de oportunidades.

Gil O, Carlos Javier. (2018). Estrategias de afrontamiento en población adulta venezolana en la ciudad de Cúcuta. identifico los estilos de afrontamientos utilizados por la población mayor de edad del hogar de paso divina providencia, en el barrio la parada, se tuvo en cuenta casos reales e historias de vida tomados por la comunidad, se analizó los estilos de afrontamiento a través de los teóricos como Lazarus \& Folkman. El estilo de afrontamiento orientado al problema, como es la emoción y el evitativo, que categoriza e identifica el afrontamiento de las personas en la investigación, siendo, el eje central la emoción, por tal manera la emoción se presentó y fue más marcada en el género femenino, ya que las mujeres tienden a afrontar los problemas, resaltando en ellas una afectación emocional, también se estudió el estrato social de la persona y se obtuvo; que entre más estrato tenga la persona menor es el estilo de afrontamiento, la cual nos dice que las personas con más estrato tienden a estancarse y no buscar una solución o herramientas inmediata para solucionar sus problemas.

Botello Pradilla Viviana Andrea; Santafé Prada Ever; Sánchez Jiménez Paola; AguilarBarreto Clara Paola; Hernández Peña Yurley Karime. (2016). Caracterización de la realidad migratoria, perspectivas y retos. Un segmento dedicado a la noción del concepto migración. El estudio permitió concluir, la migración es un fenómeno complejo, multidimensional y multifactorial que en las nuevas épocas representan una realidad que tiende al aumento, debido por la crisis económica, sociales, y políticas en la realidad del tiempo, lo países con menos población de migrantes es Honduras, Argentina y Venezuela. En el caso de Colombia tiene inmigrantes debido a la posición geográfica y cercanía a Venezuela.

Arenas Villamizar, Silva Monsalve Vivian,
Gloria Inés, Annicchiárico Lobo, Julio Humberto, Liliana Contreras Manrique, Ginny Roció Luna Rodríguez, Yadira Martínez de Biava, Valmore Bermúdez, Yanela Paola Jaimes Parada, Nathalie Raynaud. (2018). Analizarón los resultados de investigación en psicología teniendo en cuenta las contribuciones en intervención psicológica y psicoterapia de los últimos 10 años en la región del Norte de Santander; como es la comprensión del estado psicológico de los habitantes de la región, el análisis de variables psico-sociales relacionadas estado psicológico de la población nortesantandereana y se revisaron métodos terapéuticos y sus efectos en el mejoramiento de la salud mental.

\section{METODOLOGÌA}

La investigación tiene como enfoque cuantitativo siguiendo la metodología de Hernández, Fernández y Baptista (2016), "el enfoque cuantitativo utiliza la recolección y el análisis de datos para contestar preguntas de investigación y probar hipótesis previamente hechas, confía en la medición numérica, el conteo y frecuentemente en el uso de estadística para establecer con exactitud patrones de comportamiento en una población" (p.5). En todo trabajo investigativo es necesario determinar el tipo de investigación que se aplicará; se busca se puede establecer el enfoque, para Hernández; Fernández \& Baptista (2016); la investigación cuantitativo no experimental. El diseño de la investigación es descriptivo, según

Tamayo (2004) la descripción del problema con las características en la cual se está indagando, con la ayuda del registro, análisis e interpretación de la naturaleza o fenómeno presenteenlacomunidadestudiada. Esteenfoque trabaja sobre las realidades de los hechos y sus características, para interpretar y trasformar la problemática social en benefició de la población. (p. 54) . El muestreo no probabilístico se realiza por el criterio, juicio y decisión del investigador para elegir los elementos de forma subjetiva o 
criterios no basados en el azar (Hurtado 2008), se encuentran el Muestreo Intencional o por conveniencia, teniendo en cuenta criterios teóricos que de alguna manera sugieren que ciertas unidades son las más convenientes para acceder a la información que se necesita. La muestra es 100 personas venezolanas migrantes del corregimiento de la Parada, Norte de Santander y se aplicara un cuestionario de Factores Psicosociales Asociados al Bienestar de migrantes y su autoría es: Javier Murillo Muñoz y Fernando Molero.

Marroni. (2000). considero que la constitución y la consolidación de las redes migratorias, son factores de la migración. Por ello las redes tienen un efecto multiplicador en la migración de cadena; además, siendo, redes principales en las acciones migratorias (Arango 2003, Massey, et al. 1991). De hecho, su acción es acumulativa, con tendencia a crecer, al constituir cada desplazamiento un recurso para la supervivencia y superación del ser humano, en la cual se busca un buen futuro en la dinámica del crecimiento o represamiento de las redes migratorias (Arango, 2003).

Rubio (2001). Afirmo y menciono que las redes sociales son una herramienta relevante que permite que migrantes, ex migrantes y no migrantes se mantenga en comunicación e interacción con sus familiares, amigos compartiendo culturas diferentes en otros lugares, yaque la redes mantiene la comunicación y los lasos de amistad en constante interacciòn a nivel nacional e internacional para mantener los vínculos de fraternidad entre los dos países hermanos.

El enfoque de sistemas o "Teoría de los sistemas de migración" (Massey et al, 2000) se basa en los preceptos de las teorías sobre la perpetuación de los movimientos migratorios, en la cual la población migatoria se consolidan y forman su propia estructura con el paso de los tiempos y escenarios diferentes originando un sistema de migración estable según el país en la que se posesionan. $s$ vínculos de fraternidad entre los dos países hermanos.

\section{PROCEDIMIENTO Y RESULTADOS}

El estudio con los migrantes del corregimiento de la Parada en Villa Rosario. Se identificaròn las características de los factores que influyen en el bienestar de los venezolanos en la zona de frontera. Por tanto se le aplico el cuestionario que contienen los diferentes factores; el primer factor es de Prejuicio percibido, un $90 \%$ en los migrantes, han percibido ser poco agradable y se encuentra estigmatizados en la zona de frontera, seguidamente, el segundo factor, la discriminación percibida, un $99 \%$ en los migrantes, perciben la discriminación, ya que están estigmatizados como delincuentes, como personas flojas y poco trabajadoras en el tercer factor, la identidad de nacionalidad, un $98 \%$ de los migrantes reconoce ser venezolanos y se le dificulta la economía colombiana. En el cuarto factor, la satisfacción con la vida, un $45 \%$ en los migrantes, son resilientes a un nuevo proyecto de vida. El quinto factor, Autoestima, un $85 \%$ en los migrantes se encuentra con sentimientos de tristeza, ya que se encuentran lejos de sus familias, perdiendo sus propiedades para buscar un mejor porvenir y por último bienestar material percibido, un $40 \%$ en los migrantes reconoce el apoyo de Colombia y el apoyo a nivel internacional con ayudas humanitarias hacia la población migrantes. 


\section{CARACTERIZACIÒN DE LOS FACTORES PSICOSOCIALES EN LOS MIGRANTES DE LA PARADA, NORTE DE SANTANDER}

TABLA I . INFORMACIÓN GENERAL.

\begin{tabular}{|l|l|}
\hline \multicolumn{1}{|c|}{ VARIABLE } & \multicolumn{1}{c|}{ CARACTERIZACIÒN } \\
\hline Factor de Prejuicio percibido & $\begin{array}{l}\text { Percepciòn: poco agradables, hay prejuicios en contra de los } \\
\text { migrantes. } \\
\text { reciben trato negativo debido a su pertenencia grupal de } \\
\text { venezolanos. }\end{array}$ \\
\hline $\begin{array}{l}\text { Factor de la discrimi nación } \\
\text { percibida }\end{array}$ & Discriminación social, estigmatización social \\
\hline $\begin{array}{l}\text { Factor la identidad de } \\
\text { nacionalidad }\end{array}$ & $\begin{array}{l}\text { víctimas directas de experiencias como rechazo, exclusión, o } \\
\text { trato negativo. }\end{array}$ \\
\hline Factor la Satisfacción con la vida & $\begin{array}{l}\text { Persona se reconoce y acepta como miembro de la nacionalidad } \\
\text { venezolana y la }\end{array}$ \\
& valoración emocional de la procedencia. \\
\hline Factor de la Autoestima & Poca resiliencia en la parte social, laboral \\
\hline Factor bienestar material & $\begin{array}{l}\text { Amor propio, amor de familia, unión familiar: } \\
\text { Presentándose el sentimiento de tristeza y la baja de autoestima. }\end{array}$ \\
\hline Economía y sociedad: \\
vivo mucho \\
mejor económicamente en Colombia, que si viviera en \\
\hline
\end{tabular}

Fuente: Elaboración propia con base en los resultados de las encuestas. 


\section{FIGURA I. FACTORES PSICOSOCIALES EN LOS MIGRANTE DE LA PARADA, NORTE DE SANTANDER}

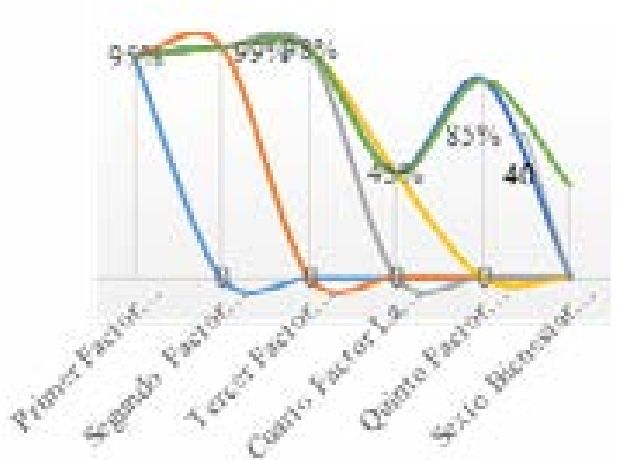

Fuente: Elaboración propia con base a los resultados a partir del cuestionario Murillo Muñoz, Javier, \&

Molero Alonso, Fernando. origen colombiano

\section{DISCUSIONES}

En los antecedentes. Gil Ortiz, Carlos Javier. (2018). Estrategias de afrontamiento en población adulta venezolana en la ciudad de Cúcuta. Tuvo como objetivo identificar los estilos de afrontamientos utilizados por la población mayor de edad del hogar de paso divina providencia, la cual se ubica en el barrio la parada en la ciudad de Cúcuta. se encontró que según mas estrato tenga la persona menor es el estilo de afrontamiento, la cual nos dice que las personas con más estrato tienden a estancarse y no buscar una solución inmediata. En la investigación; factor de la Satisfacción con la vida, un $45 \%$ en los migrantes, son resilientes en un nuevo proyecto de vida buscando dar solución a la problemática social, familiar y económica en la que atraviesan los migrantes venezolanos.

Clemens, Michael \& Cindy Huang (2019). La migración es lo que hacemos de ella. Los efectos de la migración dependen de la respuesta al dónde, cuándo, cómo y quién. El debate político sobre la migración se ha centrado en medio de las crecientes crisis de refugiados. De manera similar, la migración puede costar dinero a en la economía de un país; puede conducir a mejorar o empeorar la calidad de los servicios públicos. Por lo tanto, en el caso de la investigación; en factor de la discriminación percibida, un $99 \%$ en los migrantes, se perciben la discriminación, ya que están estigmatizados como delincuentes y en el factor de la identidad de nacionalidad, un $98 \%$ de los migrantes reconoce ser venezolanos y se le dificulta la economía colombiana.

Contreras Manrique, L., Contreras Manrique, R. d., \& Ovalle Lizcano, T. V. (2019). Asociación Fonoaudiológica, Psicológica y Odontológica en la patología del Bruxismo: en los universitarios de Cúcuta. Los síntomas de la ansiedad psíquica que se presenta en los estudiantes universitarios. De tal manera, prevalecen los síntomas psíquicos y se observa los siguientes rasgos de personalidad como la escala de ansiedad con características de preocupaciones, tensión, insomnio y estrés. Estos rasgos de personalidad, también se presentan en los migrantes venezolanos ya que se encuentra en dificultad económica, familiar y social.

Sánchez Isaza \& Cataño Ordoñez, (2016). Colombia vive hoy por hoy uno de sus más grandes problemas sociales como es la migración de familias a nivel intermunicipales e 
interdepartamentales, lo que hace necesario la caracterización del factor de riesgo total en estas familias para su intervención. La problemática que viene afectando a las poblaciones rurales, aun cuando el gobierno viene desarrollando planes direccionados hacia las familias migrantes, los problemas de infraestructura de las viviendas y la situación socioeconómica en ellos se refleja en su salud. Como consecuencia; en la investigación, en el quinto factor de la autoestima, un $85 \%$ en los migrantes se encuentra con sentimientos de tristeza, ya que se encuentran lejos de sus familias y porque perdieron sus bienes.

Ovalle Lizcano, T. V, Coronel

Peñuela, D. L., Contreras Manrique,

R. de B, \& Cabrera Reyes, A. (2019). Impacto sobre la seguridad personal frente. A la regulación de la ciberdelincuencia en la Universidad de Pamplona, sede de Villa del Rosario. Es relevante que brinden información y orientación a los educandos migrantes a través de reuniones de capacitación y a su vez, implementar estrategias grupales creando conciencia en la comunidad educativa. Este proceso de apoyo al estudiante migrante con las herramientas de las TIC facilita los procesos de enseñanza -aprendizaje para la socialización e integración y seguridad con la regulación del delito cibérnetico.

Suárez, A; Espinel, M; Contreras Manrique, L; Contreras Manrique, R. (2015). Herramientas de apoyo para el aprendizaje de los estudiantes que presentan limitaciones auditivas. Identifico las herramientas que brindan un mejor acceso al mundo del aprendizaje y en la inclusión escolar para mejorar la satisfacción personal, comunicación, interacción y los resultados académicos; logrando con esta dinámica bajar los niveles de ansiedad, sentimientos de tristeza e introversión en los pares que presentan discapacidad auditiva, visual, motora en los niños y jóvenes migrantes.

\section{PROPUESTA DE SOLUCIÓN Y RECOMENDACIONES}

Los migrantes venezolanos obtienen las ayudas económicas o humanitarias, por tal motivo, debería el departamento de migración orientarlos a que sean emprendedores para mejorar su estabilidad económica en sus familias y así mismo, se hace necesario fortalecer su autoestima para generar la resiliencia en un proyecto de vida a través de apoyo psicológico y financiero fortaleciendo el emprendimiento empresarial.

\section{CONCLUSIONES}

Los factores psicosociales que influyen en el bienestar de los migrantes, se encuentra el factor de prejuicio percibido, en este primer factor, los migrantes se perciben poco agradable ante la sociedad norte santandereana por tal motivo los perciben como personas asistencialista; seguidamente, el factor discriminación percibida, se perciben la discriminación, ya que están estigmatizados como delincuentes, facilistas y poco emprendedores; en el tercer factor la identidad de nacionalidad los migrantes reconoce ser venezolanos y con orgullo lo expresan, también se les dificulta la economía colombiana. En el cuarto factor la satisfacción con la vida, se encuentran en un porcentaje mínimo en los migrantes ya que, por la falta de oportunidad de empleo no son resilientes a un nuevo proyecto de vida y el siguiente factor de autoestima en los migrantes se presenta los sentimientos de tristeza y baja autoestima encontrarse lejos de sus familias; también es a causa por la pérdida de sus propiedades.

Finiquitando, con el factor bienestar material percibido en los migrantes reconoce un mínimo apoyo de Colombia y el apoyo a nivel internacional con ayudas humanitarias hacia la población migrantes. 


\section{REFERENCIAS BIBLIOGRÁFICAS}

Arenas Villamizar, Silva Monsalve Vivian, Gloria Inés, Annicchiárico Lobo, Julio Humberto, Liliana Contreras Manrique, Ginny Roció Luna Rodríguez, Yadira Martínez de Biava, Valmore Bermúdez, Yanela Paola Jaimes Parada, Nathalie Raynaud. (2018). Tendencias en intervención psicológica y psicoterapia en el Norte de Santander-Colombia. AVFT-Archivos Venezolanos de Farmacología y Terapéutica. Vol 37, No 5.

Arango, Joaquín (2000). Enfoques conceptuales y teóricos para explicar la migración. En: Revista Internacional de Ciencias Sociales, No 165, septiembre, pp. 3347.

Albuja, S. \& Ceballos, M. (2010). Desplazamiento urbano y migración en Colombia. Revista Migraciones Forzadas. (34), PP..10-11.

Botello Pradilla,V, Santafé Prada, E., Sánchez Jiménez, P., AguilarBarreto, C.P., y Hernández Peña, Y. (2018). Caracterización de la realidad migratoria, perspectivas y retos. En A.J. Aguilar-Barreto, V. Bermúdez-Pirela y Y.K. Hernández. (Eds.), Sociedad y derecho. (pp. 70-95). Cúcuta, Colombia: Ediciones Universidad Simón Bolívar

Camargo, Astrid León (2018). El impacto de la migración venezolana en el empleo en Colombia. Docente de la Universidad de los Llanos, Colombia. aleonc@ unillanos.edu.co

Contreras-Manrique, R. de B., ContrerasManrique,L, Ovalle-Lizcano,T.V, Espinosa-Bohórquez, L. M, ValeroBencardino, J. ., Coronel-Peñuela, D. L. ., \& Cabrera-Reyes, A. . . (2020).
Descripción de los problemas sociales influyentes en la transición moral en una sociedad cambiante a través del enfoque cuantitativo. Eco Matemático, 11(2). https://doi.org/10.22463/17948231.3016

Contreras-Manrique, L., Contreras-Manrique, R. de B., Ovalle-Lizcano, T. V, \& RincónLeal, J. F. (2020). Análisis descriptivo de rasgos de personalidad en relación con el género y la edad de los estudiantes de la universidad de pamplona en la sede villa rosario. Eco Matemático, 11(1). https://doi.org/10.22463/17948231.294.

Contreras Manrique, L., Contreras Manrique, R. d., \& Ovalle Lizcano, T. V. (2019). Asociación Fonoaudiológica, Psicológica y Odontológica en la patología del Bruxismo: En jóvenes universitarios de Cúcuta. Areté, 19 (1), [pgln]-[pgOut]. Obtenido de: https:// arete.ibero.edu.co/article/view/6586-1. DOI: 10.33881/1657-2513.art.19107

Clemens, Michael; Cindy Huang; Jimmy Graham y Kate Gough.(2019) . La migración es lo que hacemos de ella. Estudios de Política exterior.

Deusdad Ayala, B., Moya, E. M., \& Chávez Baray, S. M. (2012). Violencia de Género y Mujeres Migrantes en la Frontera: el Caso de El Paso, Texas. Portularia: Revista de Trabajo Social. Retrieved from http://rabida.uhu.es/ dspace/bitstream/handle/10272/5904/ Violencia_de_genero_y_mujeres_ migrantes. . dff?sequence $=2$.

Fernández-Niño, Julián Alfredo; Luna-Orozco, Karen; Navarro-Lechuga, Edgar; Flórez-García, Víctor; Acosta-Reyes, Jorge; Solano, Alma; Bravo, Elsa; Goenaga, Eloina: (2018). Necesidades percibidas de salud por los migrantes 
desde Venezuela en el asentamiento de Villa Caracas -Barranquilla, reporte de caso en salud públicaRevista de la Universidad Industrial de Santander. Salud, vol. 50, núm. 3, 2018Universidad Industrial de Santander, Colombia Disponible en: http://www.redalyc.org/ articulo. oa? id=343856318010DOI: https://doi.org/10.18273/revsal.v50n3$\underline{2018002}$

García Flórez, J.A. (2013). Patrones de migración en Colombia desde la perspectiva de la teoría de redes. Cuadernos de Economía, 32(59), 339-364. Retrieved from http://revistas.unal.edu.co/index. php/ceconomia/article/view/38487

Gil Ortiz, Carlos Javier. (2018). Estrategias de afrontamiento en población adulta venezolana en la ciudad de Cúcuta. https://hdl.handle. net/20.500.12442/3477

Hernández, R; Fernández, C. y Baptista, P. (2016). Metodología de la Investigación. Bogotá: McGraw Hill.

Hurtado de Barrera.J (2008). proyecto de investigación: comprensión holística de la metodología y la investigación. Editorial Quirón. Caracas, Venezuela.

Massey, Douglas S.; Arango, Joaquín; Graeme, Hugo; Kouauci, Alí; Pellegrino, Adela y Taylor, J. Eduard (1993). Theories of international migration: a review and appraisal. En: Populatión and Development Review, Vol. 19, $\mathrm{N}^{\circ} 3$, septiembre, pp. 431-466.

Massey, D. et al. (2000). Theories of Migration: A Review and Appraisal. Population and Development Review, 19 (3), 431-466.
Murillo Muñoz, Javier, \& Molero

Alonso, Fernando. (2012). Factores Psicoso-

ciales asociados al bienestar de inmigrantes de origen colombiano en. España. Psychosocial Intervention, 21(3),319329.

Ovalle Lizcano, T. V, Coronel

Peñuela, D. L., Contreras Manrique,

R. de B, \& Cabrera Reyes, A.(2019). Impacto sobre la seguridad personal frente a la regulación de la ciberdelincuencia en la Universidad de Pamplona, sede de Villa del Rosario. Respuestas, 24(3), 14-25. https://doi.org/10.22463/0122820X. 1845

Pereda Sagredo, E; V. Arnaiz

Adrián; Localización: FMC:

Formación Médica Continuada en

Atención Primaria, ISSN 1134-2072,

Vol. 17, № 2.

Pineda G, Esther. y Ávila, Keymer

(2019). La situación política, económica y social de Colombia.

Misión Jurídica: Resvista de derecho

hoy sociales, ISSN 1794-600X,

Vol.12, №. 16, 2019, págs. 59-78

Rubio, R. (2001). Redes sociales y mecanismos de apoyo en la migración de mexicanos a Estados Unidos: datos de una encuesta de flujos. Castelo Branco: Comunicación presentada al VI de la ADEH.

Sánchez Isaza, D. C., \& Cataño Ordoñez, N. (2016). Factores de riesgo total en familias migrantes en Soledad, Atlántico. Revista Cuidarte, 7(1), 1152-62. https:// doi.org/10.15649/cuidarte.v7i1.174. 
Suárez, A; Espinel, M; Contreras Manrique,

L; Contreras Manrique, R. (2015).

Herramientas de apoyo para el aprendizaje de los estudiantes que presentan limitaciones auditivas. Revista Areté, Editor Corporación Universitaria Iberoamericana, volumen (15), 1. 52-62. ISSN 1657-2513. DOI: 10.33881/16572513

Tamayo y Tamayo. (2004). El Proceso de la Investigación. México: Limusa. p. 54

Tovar Cuevas, Luis Miguel, \& Victoria Paredes, María Teresa, \& Tovar Cuevas, José Rafael, \& Troncoso Marmolejo, Gilberto, \& Pereira Laverde, Fernando (2018). Factores asociados a la probabilidad de emprendimiento en migrantes colombianos que retornan a Colombia. Migraciones Internacionales, 9(34),169192. [fecha de Consulta 6 de Octubre de 2020]. ISSN: 1665-8906. Disponible en: $\quad$ https://www.redalyc.org/articulo. oa?id=151/15160667007 